\title{
O sentimento de constrangimento: evidências acerca do contágio emocional e do gênero
}

\author{
Explaining the embarrassment feeling: emotional \\ contagion and gender evidences
}

\author{
Valdiney Veloso GOUVEIA \\ Theodore SINGELIS \\ Valeschka Martins GUERRA ${ }^{3}$ \\ Giovani Amado RIVERA ${ }^{4}$ \\ Tatiana Cristina VASCONCELOS ${ }^{4}$
}

\begin{abstract}
Resumo
O objetivo deste estudo foi saber em que medida o sentimento de constrangimento experimentado em diversas circunstâncias sociais poderia ser explicado pelo contágio emocional e gênero. Tratou-se de um estudo correlacional, com a participação de 325 pessoas, a maioria do sexo feminino (65,7\%), com idade entre 14 e 75 anos (média= 26,7; desvio-padrão= 10,40). Um terço era formado por membros da população geral e os demais eram estudantes (87,7\% universitários; $12,3 \%$ secundaristas). Todos responderam individualmente ao Questionário de Sentimento de Constrangimento, à Escala de Contágio Emocional e a um conjunto de perguntas demográficas. Os resultados indicaram que a pontuação total de constrangimento e seus fatores específicos se correlacionaram negativamente com o contágio emocional. As mulheres apresentaram maior pontuação nas dimensões de constrangimento do que os homens. Os resultados foram analisados com base nas atuais teorias acerca da emoção, considerando igualmente a perspectiva de gênero.
\end{abstract}

Palavras-chave: constrangimento; contágio emocional; gênero.

\begin{abstract}
This study aimed to comprehend the extension of emotional contagion and gender influences on the embarrassment experienced in several social circumstances. It was a correlational study, with 325 participants, 65\% of which were female, all aged from 14 to 75 years old (media $=26.7$; standard deviation $=10.4$ ). The sample group included students, $87.7 \%$ of them were from the local university, 12.3\% were high school students, and the rest of the group were from the general population. They answered individually the Embarrassment Questionnaire, the Emotional Contagion Scale, and also a set of demographic questions. Results showed that the total score of embarrassment and its specific factors are negatively correlated to the emotional contagion. Women have presented higher scores on embarrassment dimensions. These findings were analyzed based on the present theories of emotions, as well as on a gender perspective.
\end{abstract}

Key words: embarrassment; emotional contagion; gender.

vणv

1 Professor Doutor, Departamento de Psicologia. Universidade Federal da Paraíba. Campus Universitário, s/n., Castelo Branco, 58051-900, João Pessoa, PB, Brasil. Correspondência para/Correspondence to:V.V. GOUVEIA.E-mail: <vvgouveia@uol.com.br>.

2 Professor Doutor, Departamento de Psicologia, Universidade Estadual da Califórnia. Los Angeles, CA, USA.

3 Doutoranda, Departamento de Psicologia, Universidade de Kent. Canterbury, Inglaterra.

4 Professores, Universidade Federal da Paraíba. João Pessoa, PB, Brasil.

Agradecimentos: Durante a preparação deste artigo, o primeiro autor contou com bolsa de produtividade do CNPq (Proc. 520521/99-4), instituição a qual os autores agradecem. Também desejam expressar seu agradecimento à Lívia de Oliveira Borges, responsável pela coleta dos dados em Natal, e a dois revisores anônimos que apresentaram contribuições valiosas. 
De acordo com Parrott (1996), as emoções compreendem reações fisiológicas, comportamentais e cognitivas perante eventos pessoalmente significativos que se associam a sentimentos subjetivos de prazer ou desprazer. Elas desempenham papel importante na vida social dos indivíduos, permeando, favorecendo ou dificultando as relações interpessoais e a apresentação do eu.

Os estudos acerca desse construto psicológico têm procurado enfatizar a natureza e a existência de emoções básicas, o julgamento das emoções, as diferenças individuais na disposição emocional, a intensidade e a reatividade da emoção (Cacioppo \& Gardner, 1999). No caso específico das pesquisas acerca das emoções básicas, o sentimento de constrangimento (desconcerto) tem sido considerado central, especialmente no que diz respeito às relações entre as emoções e as situações sociais em que se fazem presentes, real ou imaginariamente, agentes sociais (Haidt, 2003; Rozin, Lowery, Haidt \& Imada, 1999).

Parrott (1996) afirma que o construto sentimento de constrangimento tem conseqüências evidentes no âmbito das relações interpessoais, pois está relacionado aos papéis desempenhados e às identidades sociais assumidas pelos indivíduos. Os estudos sobre o tema têm procurado enfatizar sua fonte de origem (Cupach \& Metts, 1992; Edelmann, 1987) e os fatores que podem explicar as diferenças individuais em medidas desse sentimento (Edelmann \& McCusker, 1986). Dentre tais fatores, evidenciam-se como preponderantes a suscetibilidade ao contágio emocional (Cacioppo \& Gardner, 1999; Doherty, 1997) e o gênero (Cross \& Madson, 1997; Watkins et al., 1998). No contexto brasileiro, entretanto, são escassos os estudos desse tema. Em busca realizada em dezembro de 2005 no Index Psi (www.bvs-psi.org.br), por exemplo, incluindo a palavra "constrangimento", foram encontradas 24 citações no Lilacs e sete no SciELO, porém seus resumos não revelaram qualquer relação com o tema em questão, motivando a presente pesquisa.

Especificamente em culturas coletivistas, como a brasileira (Gouveia, Albuquerque, Clemente \& Espinosa, 2002; Gouveia \& Clemente, 2000; Hofstede, 1984), o constrangimento está presente em muitos dos comportamentos e interações interpessoais, pois está relacionado à necessidade de pertencer (belonging) a um grupo e ao sentido de obrigação com os outros, característicos de seus membros (Triandis, 1995). Dessa forma, este estudo tem como objetivo avaliar em que medida o grau com que brasileiros experimentam o sentimento de constrangimento pode estar relacionado e/ou ser influenciado por sua suscetibilidade ao contágio emocional e o seu gênero. Portanto demanda-se considerar mais detalhadamente tais construtos.

\section{O sentimento de constrangimento}

Alguns estudos transculturais têm começado a mostrar que reações emocionais são freqüentemente os melhores preditores de julgamentos morais (Haidt, Koller \& Dias, 1993). As chamadas emoções morais são divididas em duas tríades: a tríade vergonha, constrangimento e culpa, e a constituída por desprezo, raiva e repugnância - essa última denominada como tríade da hostilidade (Larrington, 2001).

Para Haidt (2003), as emoções morais são aquelas ligadas aos interesses ou ao bem-estar da sociedade como um todo, assim como ao bem-estar do outro (não-eu), não envolvido diretamente no comportamento em questão, como juízes ou agentes. As emoções que constituem o primeiro pólo são consideradas centradas no eu e, algumas vezes, são denominadas de autoconscientes. Ainda de acordo com Haidt (2003), essas emoções estão totalmente relacionadas a aspectos da vida social que podem variar culturalmente (por exemplo, a ênfase em uma estrutura social hierárquica versus igualitária, característica de sociedades coletivistas verticais e horizontais, respectivamente (Rozin et al., 1999; Triandis, 1995) sugerem que tais emoções são cruciais para a civilização humana, pois refletem ou implementam a internalização da ordem social no indivíduo.

Segundo Schlenker e Leary (1982), o constrangimento é definido como uma experiência ou estado emocional, expresso como um tipo de ansiedade social e que resulta da perspectiva da avaliação dos outros em situações reais ou imaginárias. Esse sentimento compreende uma espécie de reação psicológica ao comportamento de contrariar as normas e demandas sociais, obedecendo ao desejo de agir segundo as expectativas e interesses dos demais (Singelis \& Sharkey, 1995). 
Parrott (1996) afirma que o constrangimento é a mais social de todas as emoções e que requer um reconhecimento das convenções sociais, assim como uma representação das crenças e avaliações dos outros. Em geral, segundo esse autor, as pessoas procuram evitar esse sentimento, pois a experiência emocional envolve uma sensação de inaptidão social ou imprudência, associada à surpresa. Fisicamente, tal reação é acompanhada por sinais evidentes de nervosismo, como rubor e redução do contato visual. No entanto, apesar de o termo constrangimento ser, muitas vezes, confundido com o sentimento de vergonha, Sabini, Garvey e Hall (2001) procuraram diferenciar esses dois construtos em função dos seus antecedentes, de forma a garantir a existência do sentimento de constrangimento como uma emoção distinta (Edelmann, 1987).

Segundo Keltner e Buswell (1997), seus antecedentes estão relacionados a situações e comportamentos que resultam de uma violação das convenções ou normas sociais, implicando um aumento da exposição social do indivíduo. As experiências constrangedoras mais comumente reportadas são situações gerais de confusão: física e de controle corporal (tropeçar em algo), cognitiva (esquecer algo importante), manutenção de privacidade (ter seus sentimentos expostos) e interações sociais desastrosas. Todas essas situações são consideradas constrangedoras, pois envolvem convenções sociais que, ao serem violadas, levam a uma avaliação negativa do comportamento e da identidade do ator.

Keltner e Buswell (1997) afirmam que para surgir o constrangimento é necessária a ação de processos cognitivos complexos, como realizar uma avaliação a partir da perspectiva de outra pessoa, fazendo com que esse sentimento se manifeste mais tardiamente no desenvolvimento do ser humano. Nesse sentido, Edelmann (1987) mostrou que o constrangimento aumenta com a faixa etária, a partir da infância tardia.

Apesar de a definição do construto constrangimento obter certo consenso, tem sido bastante debatida a questão de sua operacionalização, devido, principalmente, às dúvidas acerca da sua dimensionalidade. Edelmann (1987) reúne provas a favor de uma estrutura unidimensional, porém os resultados de Miller (1992) permitem pensar que existem mais dimensões, correspondendo às circunstâncias sociais que provocam esse sentimento. É nos resultados encontrados por esse último autor que este estudo está baseado.

Miller (1992) agrupa as situações sociais em quatro grupos principais, a saber: (1) comportamento individual, subdividido em deficiência pública normativa, constrangimento por prejudicar a outro e ser o centro das atenções; (2) comportamento interativo, expresso como interação desajeitada e constrangimento do grupo a que a pessoa pertence; (3) provocações dos demais, que se referem às transgressões reais e/ou irreais; e (4) comportamento de espectador, definido como constrangimento empático.

Theodore Singelis procurou operacionalizar essas categorias de Miller (1992), propondo uma medida de constrangimento com oito fatores: deficiência pública normativa, falha em regular a privacidade do outro, falha em regular a própria privacidade, situação de protagonista, perda de papel, sentir-se culpável, desconcerto endogrupal e desconcerto empático. Entretanto, no Brasil, Gouveia, Singelis, Guerra, Santos e Vasconcelos (2005) observaram que o conjunto desses fatores converge para uma pontuação total de constrangimento.

De acordo com a denominação dos fatores da medida de constrangimento, eles não envolvem apenas situações sociais vivenciadas pelo indivíduo em interação real ou imaginária, mas também por outras pessoas. Para Miller (1987) as respostas emocionais perante as experiências de outras pessoas são claramente afetadas pela perspectiva do observador perante o outro, pela avaliação cognitiva desses outros e pela identificação com eles. Tem-se comprovado que as pessoas mais sensíveis à visão dos demais são particularmente ansiosas em relação a sua própria imagem pública, dando ênfase em atributos internos do eu e demonstrando maior propensão a experimentar constrangimento (Singelis \& Sharkey, 1995).

Assim, de acordo com Miller (1987), quando uma pessoa está em uma situação constrangedora, os observadores podem reconhecer e, de forma empática, compartilhar aquele constrangimento, mesmo que as ações da pessoa não reflitam nem ameacem a identidade social do observador. Essa hipótese foi testada pelo autor e demonstrou uma correlação de $r=0,36$ 
$(p<0,05)$ entre a percepção do constrangimento do ator e o constrangimento relatado pelo observador. Observadores com altas pontuações em constrangimento consideraram os atores como mais constrangidos, reportaram maior constrangimento empático e exibiram maior quantidade de reações fisiológicas do que os observadores com menores pontuações em constrangimento. Nesse sentido, um fator que pode ter um impacto fundamental no desencadeamento do sentimento de constrangimento, principalmente do constrangimento empático, é a suscetibilidade ao contágio emocional (Doherty, 1997), construto que será discutido a seguir.

\section{Contágio emocional}

Como emoção, o constrangimento afeta momentaneamente a identidade apresentada pelo indivíduo na interação social. Essa identidade, estável ou flexível, pode ser ameaçada por um outro elemento inerente ao sujeito, denominado de contágio emocional. De acordo com Doherty (1997), as pessoas tendem a "imitar" inconscientemente as expressões emocionais do outro, o que produz uma experiência emocional simultânea e congruente com a original. Assim, a suscetibilidade individual a um maior contágio emocional já foi relacionada à genética, ao gênero, às experiências prévias e características de personalidade (Cacioppo \& Gardner, 1999; Doherty, 1997).

Segundo Doherty (1997), as pessoas especialmente suscetíveis são aquelas: (a) que prestam muita atenção e são capazes de ler as expressões emocionais dos outros; (b) que se percebem como interdependentes e inter-relacionadas, muito mais do que independentes e únicas; (c) que tendem a imitar expressões faciais, vocais e posturais; e (d) cuja experiência emocional consciente é muito influenciada por feedbacks periféricos, isto é, comentários e observações realizadas a seu respeito por outros, ainda que de forma indireta.

Barsade (2002) procura diferenciar o contágio emocional do contágio cognitivo. Para o autor, a transferência de idéias é qualitativamente diferente da transferência de sentimentos. Para a compreensão de idéias, os objetos centrais são as palavras, que, no entanto, são consideravelmente menos importantes na compreensão das emoções, nas quais dicas não verbais são essenciais. Devido à importância dada a essas expressões não verbais, o contato interpessoal direto é fundamental para a transmissão de emoções. Portanto o contágio emocional depende muito mais de expressões corporais e não verbais.

Em situações de grupo, a expressão das emoções é percebida pelos seus membros por meio de sinais não verbais, como expressões faciais, linguagem corporal e tom de voz. Barsade (2002) afirma que o grau de ocorrência de um contágio emocional será mediado por processos de atenção, ocorrendo maior suscetibilidade ao contágio quanto mais atenção for direcionada ao objeto. Esses processos de atenção, por sua vez, podem ser influenciados por fatores externos, como o tipo de emoção e a intensidade em que ela é expressa; por diferenças individuais como o sexo, as diferenças na tendência a imitação espontânea e uma suscetibilidade geral a apreender as emoções dos outros (Barsade, 2002; Doherty, 1997).

Nesse sentido, o contágio emocional se refere a um estado emocional no observador como resultado direto da percepção do estado emocional de uma outra pessoa (objeto de observação), sendo a intensidade dessa emoção considerada elevada e autodirigida. Preston e Waal (2002) procuram diferenciar esse sentimento de outros, como empatia, empatia cognitiva e simpatia, nos quais a emoção não é autodirigida, mas dirigida ao outro, e sugerem que tais emoções existam em um continuum no qual, em cada situação específica, localizam-se diferentes níveis de excitação, de objetos e de motivação para ajudar.

Doherty (1997) verificou em um de seus estudos que as pessoas com maior suscetibilidade ao contágio emocional são mais propensas ao constrangimento $(r=0,25, p<0,05)$, e se sentem ansiosas perante grandes grupos $(r=0,32, p<0,01)$. As maiores pontuações em contágio emocional estão positivamente associadas com emocionalidade $(r=0,29, p<0,05)$ e sensibilidade $(r=0,42, p<0,05)$. Esse construto também se correlacionou negativamente com estabilidade emocional $(r=-0,30, p<0,05)$.

Esses aspectos demonstram a ligação desse construto com características socialmente determinadas como femininas (Baron-Cohen, 2004), o que sugere uma forte influência do gênero tanto na suscetibilidade ao contágio emocional como no sentimento de constrangimento. Tais aspectos precisam ser minuciosamente considerados. 


\section{O gênero como variável sociocultural}

De acordo com Markus e Kitayama (1991), a cultura pode ter um papel central na formação da experiência emocional, pois as emoções estruturam-se de acordo com a forma com que a pessoa compreende o mundo. Na história da humanidade, a sociedade tem oferecido crenças e ideologias sobre os gêneros masculino e feminino, que apóiam e legitimam os papéis que devem assumir homens e mulheres.

Uma grande quantidade de pesquisadores tem procurado utilizar a variável gênero em seus estudos, como, por exemplo, nas diferenças existentes entre homens e mulheres nas pontuações de autoconceito (Watkins et al., 1998). Nessa pesquisa transcultural, os autores encontraram diferenças de gênero de acordo com o autoconceito e a auto-estima em culturas como as da Austrália, Canadá, Nova Zelândia, Estados Unidos e o grupo étnico branco da África do Sul, onde as mulheres enfatizaram os valores familiares e relacionamentos sociais mais do que os homens.

Baron-Cohen (2004), em seu livro "A diferença essencial", procura demonstrar sua hipótese de que a diferença entre os gêneros se deve ao tipo de cérebro de cada um: os homens têm um cérebro sistematizador, enquanto as mulheres apresentam um cérebro empatizador. Essa diferença faz com que elas sejam mais preocupadas com as relações interpessoais, tenham mais facilidade para"ler a mente"dos demais e, portanto, sejam em princípio mais suscetíveis ao contágio emocional e, em maior medida do que os homens, tendam a se constranger em situações sociais desastrosas.

Algoe, Buswell e DeLamater (2000) realizaram uma revisão de pesquisas acerca das diferenças de gênero nas emoções e concluíram que a população geral acredita na existência de uma diferença na freqüência da experiência e expressão de determinadas emoções por parte de homens e mulheres. Eles também afirmam que algumas emoções são, de fato, vivenciadas e expressas com maior freqüência por mulheres; são as chamadas emoções femininas, que incluem felicidade, vergonha, medo e constrangimento. Outras emoções são vivenciadas mais freqüentemente por homens: orgulho, desprezo e raiva. Eles categorizam ainda as emoções neutras, não relacionadas ao gênero, que incluem a repugnância, o interesse e o ciúme. Com base nesse marco teórico, esses autores procuraram estudar as diferenças de gênero com relação à interpretação da expressão facial de determinadas emoções (por exemplo: raiva, medo, repugnância, desprezo e constrangimento). Em seu estudo, eles encontraram que os participantes do sexo feminino apresentaram maior tendência a usar estereótipos de gênero com maior freqüência do que os homens na interpretação da expressão facial dos indivíduos observados. Os resultados comprovaram os efeitos do gênero na interpretação da emoção.

Em um outro estudo, realizado por Grossman, Wilhelm, Kawachi e Sparrow (2001), que compara homens e mulheres com altas pontuações de fobia social em uma situação de exposição pública em forma de discurso, foi encontrado que as mulheres demonstraram maior nível de ansiedade e constrangimento durante o discurso do que os homens. Nesse mesmo sentido, Miller (1987), ao observar as médias do constrangimento empático de acordo com o gênero, encontrou que as mulheres $(M=4,4)$ normalmente reportam maior constrangimento pessoal do que os homens $(M=3,5), F(1,58)=6,19, p<0,05$.

Sabe-se, portanto, que a sociedade está impregnada de ideologias que servem, de certa forma, como guia e justificativa das condutas do indivíduo, legitimando e contribuindo para que homens e mulheres adotem certos esquemas psicológicos, biológicos e de condutas a respeito da formação dos papéis sexuais (Baron-Cohen, 2004; Paéz, Torres \& Echebarría, 1990). É nesse contexto de desigualdade social da cultura ocidental contemporânea que se inserem as situações e interações sociais antecedentes aos construtos aqui estudados. Esta pesquisa especificamente trata de conhecer em que medida o gênero feminino é mais susceptível ou exposto à influência de circunstâncias que produzem constrangimento.

\section{Método}

Este estudo é de tipo correlacional. A variável critério corresponde ao sentimento de constrangimento, figurando como variáveis antecedentes a suscetibilidade ao contágio emocional e o gênero. Duas hipóteses principais foram formuladas: hipótese 1: a suscetibilidade ao contágio emocional estará diretamente correlacionada ao sentimento de constran- 
gimento; hipótese 2: as mulheres apresentarão maior pontuação em sentimento de constrangimento do que os homens.

\section{Participantes}

Participaram do estudo 325 pessoas, das quais 110 viviam em João Pessoa e 215 em Natal. Aproximadamente um terço delas era da população geral $(28,2 \%)$, sendo os $71,8 \%$ restantes divididos entre estudantes universitários (87,7\%) e secundaristas (12,3\%). A maioria era do sexo feminino (65,7\%), com idade entre 14 e 75 anos $(M=26,7 ; D P=10,40)$. Entre os que disseram trabalhar, 57,0\% tinham uma profissão qualificada (engenheiro, veterinário, economista, etc.). Essa amostra é intencional, portanto não probabilística; participaram aquelas pessoas que estavam presentes nas salas de aula visitadas ou que, a pedido dos pesquisadores, concordaram em responder à escala em suas próprias residências.

\section{Instrumentos}

Os participantes responderam aos seguintes instrumentos, dispostos na ordem em que se apresentam:

- Questionário de Sentimento de Constrangimento: descreve 40 situações do cotidiano para as quais o participante deve dizer em que medida as considera constrangedoras. Os itens são respondidos por meio de uma escala de sete pontos, que vai de $1=$ nada constrangedora a $7=$ muito constrangedora. Gouveia, Singelis, Guerra, Santos \& Vasconcelos (2005) comprovaram sua validade de construto no Brasil, indicando que ela cobre oito fatores do sentimento de constrangimento: deficiência pública normativa (Alfa=0,77; por exemplo: derramar uma bebida sobre si mesmo em uma festa), falha em regular a privacidade do outro (Alfa= 0,81; por exemplo: surpreender uma pessoa no provador de uma loja em roupas íntimas), falha em regular a própria privacidade (Alfa=0,80; por exemplo: ser surpreendido ao sair da ducha por um convidado da casa), situação de protagonista (Alfa =0,72; por exemplo: um grupo de amigos Ihe canta "parabéns para você" em um restaurante local), perda de papel (Alfa =0,62; por exemplo: contar uma piada a um grupo de conhecidos e, no final, ninguém sorrir), sentir-se culpável (Alfa=0,68; por exemplo: ver a namorada do seu melhor amigo beijando outro e, no dia seguinte, encontrar esse amigo), desconcerto endogrupal (Alfa=0,63; por exemplo: ir a um restaurante com amigos que soltam piadas grosseiras ao garçom e, ao retornar ao local, o garçom comentar sobre a grosseria de seu grupo de amigos) e desconcerto empático ( $\mathrm{Alfa}=0,75$; por exemplo: estar assistindo a um espetáculo amador em que o artista procura, inutilmente, fazer com que as pessoas sorriam). A pontuação total, correspondendo ao somatório de todos os itens, apresentou um Alfa (de Cronbach) de 0,92.

- Escala de Contágio Emocional: desenvolvida por Doherty (1997), é uma medida unifatorial composta de 18 itens que descrevem cinco sentimentos básicos: amor, felicidade, medo, raiva e tristeza; a escala também inclui uma sexta faceta, que procura verificar o nível de atenção dada às emoções das outras pessoas. Esses itens são respondidos em uma escala de quatro pontos, sendo $1=$ sempre e $4=$ nunca; quanto menor a pontuação, maior a suscetibilidade ao contágio emocional do participante. Sua consistência interna (Alfa de Cronbach) no estudo original foi 0,90. A adaptação dessa medida para o Brasil foi realizada por Gouveia, Guerra, Santos, Rivera e Singelis (2006), confirmando sua estrutura unifatorial (Alfa =0,82). Na presente amostra, o índice de consistência interna foi de 0,81, corroborando-se tal estrutura. Nesse caso, as saturações variaram de 0,31 a 0,64, explicando 25\% da variância total (eigenvalue de 4,50).

Finalmente, antes de dar início à participação no estudo, as pessoas consultadas deveriam expressar seu acordo assinando um termo de "livre consentimento", disposto em uma folha anexa. Na última página, figuravam ainda perguntas de caráter sociodemográfico (gênero, idade, escolaridade e profissão).

\section{Procedimentos}

A maioria dos participantes respondeu aos questionários individualmente, porém em ambiente coletivo de sala de aula. Uma vez obtida a autorização do professor da disciplina, os aplicadores se apresentavam solicitando a colaboração voluntária dos estudantes presentes. Foi-Ihes informado que se tratava de uma pesquisa sobre diferenças individuais nas atitudes e sentimento de desconcerto, não havendo respostas certas ou erradas. A todos foi assegurado que suas respostas seriam confidenciais, devendo ser tratadas 
estatística e coletivamente. Procurou-se igualmente indicar dois endereços institucionais onde os participantes poderiam se dirigir em caso de dúvidas e/ou com o fim de obter informações sobre os resultados da pesquisa. Em média, 25 minutos foram suficientes para concluir a participação. Alternativamente, uma menor quantidade de participantes, menos de 30\% do total, respondeu o questionário em sua casa, a pedido dos estudantes que previamente participaram do estudo e concordaram em solicitar que outros também o respondessem.

\section{Resultados}

Inicialmente, procurou-se conhecer o nível de contágio emocional apresentado pelos participantes do estudo, comparando-o com o ponto mediano da escala de resposta $(2,5$; amplitude de $1=$ sempre e $4=$ nunca). É importante enfatizar que quanto menor a pontuação, maior a suscetibilidade ao contágio emocional. A média dos participantes na escala, isto é, a divisão do somatório pelo número de itens (18), foi de $1,87(\mathrm{DP}=0,38)$. Esse valor é estatisticamente menor que o ponto mediano da escala [ $t(298)=28,45, p<0,001]$, indicando sua tendência a um maior contágio emocional.

A primeira hipótese afirmava que os participantes que apresentassem uma maior suscetibilidade ao contágio emocional (ou seja, uma menor pontuação nessa escala) também apresentariam uma maior tendência a se sentirem constrangidos, o que foi corroborado. A pontuação total de constrangimento correlacionou-se negativamente com a suscetibilidade ao contágio emocional $(r=-0,29 ; p<0,001)$. Os fatores específicos de constrangimento apresentaram esse mesmo padrão de correlação, como segue: desconcerto endogrupal $(r=-0,30 ; p<0,001)$, desconcerto empático $(r=-0,28 ; p<0,001)$, sentir-se culpável $(r=-0,28 ; p<0,001)$, deficiência pública normativa $(r=-0,27 ; p<0,001)$, regular a privacidade do outro $(r=-0,20 ; p<0,01)$, regular a própria privacidade $(r=-0,20 ; p<0,01)$, perda de papel $(r=-0,18$; $p<0,01)$ e situação de protagonista $(r=-0,11 ; p<0,05)$.

A segunda hipótese procurava estabelecer a relação entre o sentimento de constrangimento e o gênero, afirmando que as mulheres apresentariam uma maior pontuação no sentimento de constrangimento. Inicialmente, procurou-se verificar a diferença entre homens e mulheres no que diz respeito à pontuação total de constrangimento, tendo-se confirmado essa hipótese. Especificamente, as mulheres apresentaram uma média global $(M=4,9, D P=0,80)$ superior a dos homens $(M=4,2, D P=0,96), t(290)=6,83 ; p<0,001$.

Posteriormente, complementando os achados antes descritos, procurou-se comparar os múltiplos fatores específicos que provocam constrangimento em razão do sexo do participante. Nesse caso, efetuou-se uma Multivariate Analysis of Variance (Manova) (Tabela 1).

A Manova apresentou efeito principal do gênero nas pontuações dos fatores de constrangimento, Lambda de Wilks=0,74, $F(8,283)=12,37, p<0,001$. Coerentemente com a hipótese proposta, as mulheres apresentaram médias superiores às dos homens em sete dos oito fatores (Tabela 1). O fator situação de protagonista não apresentou diferença significativa entre os sexos $(p=0,07)$.

Tabela 1. Análise multivariada (Manova) das circunstâncias sociais que provocam constrangimento de acordo com o gênero, com as respectivas médias (M) e desvios-padrão (DP)

\begin{tabular}{|c|c|c|c|c|c|c|}
\hline \multirow{3}{*}{ Constrangimento } & \multicolumn{4}{|c|}{ Gênero } & \multirow{2}{*}{\multicolumn{2}{|c|}{ Contraste }} \\
\hline & \multicolumn{2}{|c|}{ Masculino } & \multicolumn{2}{|c|}{ Feminino } & & \\
\hline & $M$ & $\mathrm{DP}$ & M & DP & $\mathrm{F}$ & $p$ \\
\hline 1. Deficiência pub. norativa & 4,8 & 1,30 & 5,8 & 0,90 & 60,754 & 0,001 \\
\hline 2. Privacidade do outro & 4,3 & 1,44 & 5,2 & 1,26 & 31,546 & 0,001 \\
\hline 3. Própria privacidade & 4,0 & 1,52 & 5,2 & 1,14 & 60,008 & 0,001 \\
\hline 4. Situação do protagonista & 3,5 & 1,38 & 3,8 & 1,39 & 3,273 & 0,070 \\
\hline 5. Perda de papel & 4,5 & 1,15 & 4,9 & 1,03 & 10,996 & 0,001 \\
\hline 6. Sentir-se culpável & 4,5 & 1,10 & 5,3 & 1,02 & 31,684 & 0,001 \\
\hline 7. Desconcerto endogrupal & 4,2 & 1,18 & 4,8 & 1,02 & 19,957 & 0,001 \\
\hline 8. Desconcerto empático & 3,3 & 1,42 & 3,8 & 1,28 & 9,242 & 0,010 \\
\hline
\end{tabular}




\section{Discussão}

Este estudo teve como objetivo principal conhecer a relação entre o sentimento de constrangimento, a suscetibilidade ao contágio emocional e o gênero no contexto brasileiro. Espera-se que esse objetivo tenha sido alcançado. Entretanto não é possível deixar de reconhecer a especificidade da amostra tratada. Não compreendeu uma amostra probabilística, mas intencional, pois se contou com a participação daqueles que concordaram em colaborar no estudo. Além disso, majoritariamente, os participantes eram estudantes universitários; aqueles da população geral foram contatados por meio deles, o que pode implicar um grupo seletivo de pessoas, provavelmente de classe socioeconômica média-alta. Deve-se, entretanto, ressaltar que o propósito último da pesquisa não foi generalizar os resultados, mas comprovar relações que seriam teoricamente esperadas. Feitos esses comentários, cabe considerar mais pormenorizadamente os resultados antes descritos.

Os achados reforçam a relação entre a suscetibilidade ao contágio emocional e o sentimento de constrangimento (Doherty, 1997). Especificamente, foi possível observar que os participantes que apresentaram maior suscetibilidade ao contágio emocional obtiveram também maior pontuação em constrangimento, sendo essa relação especialmente verdadeira para os fatores deficiência pública normativa, sentir-se culpável, desconcerto endogrupal, desconcerto empático. Essas dimensões podem ter apresentado maiores correlações devido à sua natureza interacional e coletiva (Miller, 1992), na qual se supõe um maior nível de contato interpessoal e, conseqüentemente, uma maior possibilidade de compartilhamento de emoções.

Os resultados mostram, portanto, que os indivíduos caracterizados como sendo mais suscetíveis aos demais, que prestam muita atenção nas experiências emocionais das outras pessoas e que são influenciados pelas interações sociais se apresentaram como mais prováveis a vivenciar o sentimento de constrangimento (Doherty, 1997; Miller, 1987; Singelis \& Sharkey, 1995). É importante enfatizar, no entanto, que nenhuma dessas características representa um déficit, mas apenas reflete diferentes formas de lidar com o mundo e de interagir com os demais, que podem, em parte, resultar de contextos culturais diferenciados.
Sharkey e Singelis (1995) afirmam que uma maior sensibilidade ao contexto social e o constrangimento associado a essa sensibilidade podem ser vistos como características positivas que contribuem para a adaptação do indivíduo ao contexto e ao sistema social, especialmente nas culturas coletivistas.

Essa influência da cultura na vivência e expressão das emoções pode ser facilmente identificada pela variável gênero, que demonstrou estar relacionada com o sentimento de constrangimento, como previsto (Algoe et al., 2000; Grossman, Wilhelm, Kawachi \& Sparrow, 2001; Miller, 1987). De forma consistente com os estudos prévios, as mulheres apresentaram uma maior tendência a vivenciar o sentimento de constrangimento do que os homens. É possível que esses resultados sejam devidos aos papéis sociais construídos e atribuídos às mulheres (Belo, 2003; Oliveira, 2000), que enfatizam determinados tipos de emoção como característicos desse sexo. Não obstante há que se reportar igualmente à maior capacidade das mulheres em reconhecer e vivenciar as emoções do outro (Baron-Cohen, 2004).

Finalmente, é importante enfatizar a dificuldade para encontrar no contexto brasileiro pesquisas acerca do constrangimento, assim como de emoções básicas. Estudos sobre o tema devem ser estimulados. De acordo com Larrington (2001), algumas pesquisas da Psicologia transcultural sugerem que a emoção em si é uma base para o comportamento moral e social. Segundo Rozin et al. (1999), o mundo moral dos indivíduos não diz respeito apenas à racionalidade, mas também envolve sentimentos fortes associados aos aspectos cognitivos da moralidade. Para esses autores as emoções constituem um tipo particular de percepção ou racionalidade. Nesse sentido, o estudo que ora se apresenta compreende uma contribuição em tal direção, sendo recomendadas ainda pesquisas futuras de forma a avaliar a extensão desses achados.

\section{Referências}

Algoe, S., Buswell, B., \& DeLamater, J. (2000). Gender and job status as contextual cues for the interpretation of facial expression of emotion. Sex Roles, 42 (3-4), 183-208.

Baron-Cohen, S. (2004). Diferença essencial: a verdade sobreo cérebro de homens e mulheres. Rio de Janeiro: Editora Objetiva.

Barsade, S. (2002). The ripple effect: Emotional contagion and its influence on group behavior. Administrative Science Quarterly, 47 (4), 644-675. 
Belo, R. P. (2003). A base social das relações de gênero: explicando o ciúme romântico através do sexismo ambivalente e dos valores humanos básicos. Dissertação de mestrado não-publicada, Universidade Federal da Paraíba, João Pessoa.

Cacioppo, J. T., \& Gardner, W. L. (1999). Emotion. Annual Review Psychology, 50, 191-214.

Cross, S., \& Madson, L. (1997). Models of the self: Self-construals and gender. Psychological Bulletin, 122 (1), 5-37.

Cupach, W. R., \& Metts, S. (1992). The effects of type of predicaments and embarrassability on remedial responses to embarrassing situations. Communication Quarterly, 40 (2), 149-161.

Doherty, R. (1997). The Emotional Contagion Scale: A measure of individual differences. Journal of Nonverbal Behavior, 21 (2), 131-154.

Edelmann, R. J. (1987). The psychology of embarrassment. Chichester, UK: Wiley.

Edelmann, R. J., \& McCusker, G. (1986). Introversion, neuroticism, empathy and embarrassability. Personality and Individual Differences, 7 (2), 133-140.

Gouveia, V. V., \& Clemente, M. (2000). O individualismocoletivismo no Brasil e na Espanha: Correlatos sóciodemográficos. Estudos de Psicologia, Natal, 5 (2), 317-346.

Gouveia, V.V., Albuquerque, F. J. B., Clemente, M., \& Espinosa, P. (2002). Human values and social identities: A study in two collectivist cultures. International Journal of Psychology, 37 (6), 333-342.

Gouveia, V.V., Singelis, T., Guerra, V., Santos, W., \& Vasconcelos, T. (2005). Auto-imagem e sentimento de constrangimento. Psico, 36 (3), 231-241.

Gouveia, V. V., Guerra, V. M., Santos, W. S., Rivera, G. A., \& Singelis, T. M. (2006). Escala de Contágio Emocional: Adaptação ao contexto brasileiro. Manuscrito submetido à publicação.

Grossman, P., Wilhelm, F., Kawachi, I., \& Sparrow, D. (2001). Gender differences in psychophysiological responses to speech stress among older social phobics: Congruence and incongruence between self-evaluative and cardiovascular reactions. Psychosomatic Medicine, 63 (5),765-777.

Haidt, J. (2003). The moral emotions. In R. J. Davidson, K. R. Scherer \& H. H. Goldsmith (Eds.), Handbook of affective sciences (pp.852-870). Oxford: Oxford University Press.

Haidt, J., Koller, S., \& Dias, M. (1993). Affect, culture and morality, or is it wrong to eat your dog? Journal of Personality and Social Psychology, 65 (4), 613-628.

Hofstede, G. (1984). Culture's consequences: International differences in work-related values. Thousand Oaks, CA: Sage Publications.

Keltner, D., \& Buswell, B. (1997). Embarrassment: Its distinct form and appeasement Functions. Psychological Bulletin, $122(3), 250-270$

Larrington, C. (2001). The psychology of emotion and study of the medieval period. Early Medieval Europe, 10 (2), 251-256.

Markus, H. R., \& Kitayama, S. (1991). Culture and the self: Implications for cognition, emotion and motivation. Psychological Review, 98 (2), 224-253.
Miller, R. S. (1987). Empathic embarrassment: Situational and personal determinants of reactions to embarrassment of another. Journal of Personality and Social Psychology, 53 (6), 1061-1069.

Miller, R. S. (1992). The nature and severity of self-reported embarrassing circumstances. Personality and Social Psychology Bulletin, 18 (2), 190-198.

Oliveira, I. C. (2000). Multiplicidade de papéis da mulher e os seus efeitos para o bem-estar psicológico. Dissertação de mestrado não-publicada, Universidade Federal da Paraíba, João Pessoa.

Paéz, D., Torres, B., \& Echebarría, A. (1990). Esquema de si, representación social y estereotipo sexual. In G. Musitu (Org.), Procesos psicosociales básicos (pp.229-234). Barcelona:PPU.

Parrott, E. G. (1996). Embarrassment. In A. S. Manstead \& M. Hewstone (Eds.), The Blackwell encyclopedia of social psychology (pp.196-198). Oxford: Blackwell Publishers.

Preston, S. D., \& De Waal, F. B. M. (2002). The communication of emotions and the possibility of empathy in animals. In S. G. Post, L. G. Underwood, J. P. Schloss \& W. B. Hurlbut (Eds.), Altruism and altruistic love (pp.284-308). New York: Oxford University Press.

Rozin, P. Lowery, L., Haidt, J., \& Imada, S. (1999). The CAD triad hypothesis: A mapping between three moral emotions (contempt, anger, disgust) and three moral codes (community, autonomy, divinity). Journal of Personality and Social Psychology, 76 (4), 574-586.

Sabini, J., Garvey, B., \& Hall, A. (2001). Shame and embarrassment revisited. Personality and Social Psychology Bulletin, 27 (1), 104-117.

Schlenker, B. R., \& Leary, M. R. (1982). Social anxiety and self-presentation: A conceptualization and model. Psychological Bulletin, 92 (3), 641-669.

Sharkey, W., \& Singelis, T. (1995). Embarrassability and self-construal: A theoretical integration. Personality and Individual Differences, 19 (6), 919-926.

Singelis, T. (1994). The measurement of independent and interdependent self-construals. Personality and Social Psychology Bulletin, 20 (5), 580-591.

Singelis, T., \& Sharkey, W. (1995). Culture, self-construal, and embarrassability. Journal of Cross-Cultural Psychology, 26 (6), 622-644

Triandis, H. (1995). Individualism and collectivism. Boulder, CO: Westview Press

Watkins, D., Akande, A., Fleming, J., Ismail, M., Lefner, K., Regmi, M., Watson, S., Yu, J., Adair, J., Cheng, C., Gerong, A., Mclnerney, D., Mpofu, E., Singh-Sengupta, S., \& Wondimu, H. (1998). Cultural dimensions, gender and the nature of self-concept: A fourteen-country study. International Journal of Psychology, 33 (1), 17-31.

Recebido em: 23/6/2005

Versão final reapresentada em: 13/6/2006

Aprovado em: 28/6/2006 
